\title{
Recommended Amount of Sleep for a Healthy Adult: A Joint Consensus Statement of the American Academy of Sleep Medicine and Sleep Research Society
}

Consensus Conference Panel: Nathaniel F. Watson, MD, MSc, Moderator ${ }^{1}$; M. Safwan Badr, MD²; Gregory Belenky, MD³; Donald L. Bliwise, PhD; Orfeu M. Buxton, PhD ${ }^{5}$; Daniel Buysse, MD; David F. Dinges, PhD; James Gangwisch, PhD; ; Michael A. Grandner, PhD, MSTR, CBSM7; Clete Kushida, MD, PhD ${ }^{9}$; Raman K. Malhotra, MD ${ }^{10}$; Jennifer L. Martin, PhD ${ }^{11}$; Sanjay R. Patel, MD, MSc' ${ }^{12}$; Stuart F. Quan, MD ${ }^{12}$; Esra Tasali, MD ${ }^{13}$ Non-Participating Observers: Michael Twery, PhD ${ }^{14, *}$; Janet B. Croft, PhD ${ }^{15, *}$; Elise Maher, RPSGT ${ }^{16, *}$

American Academy of Sleep Medicine Staff: Jerome A. Barrett ${ }^{17}$; Sherene M. Thomas, PhD ${ }^{17}$; Jonathan L. Heald, MA ${ }^{17}$

${ }^{1}$ University of Washington, Seattle, WA; ${ }^{2}$ Wayne State University, Detroit, MI; ${ }^{3}$ Washington State University, Spokane, WA; ${ }^{4}$ Emory University, Atlanta, GA; ${ }^{5}$ Pennsylvania State University, University Park, PA; ${ }^{6}$ University of Pittsburgh, Pittsburgh, PA; ${ }^{7}$ University of Pennsylvania, Philadelphia, PA; ${ }^{8}$ Columbia University, New York, NY; ${ }^{9}$ Stanford University, Stanford, CA; ${ }^{10}$ Saint Louis University, St. Louis, MO; ${ }^{11}$ University of California, Los Angeles, Los Angeles, CA; $;{ }^{12}$ Harvard Medical School, Boston, MA; ${ }^{13}$ The University of Chicago, Chicago, IL; ${ }^{14}$ National Heart, Lung, Blood Institute, NIH, Bethesda, MD; ${ }^{15}$ Centers for Disease Control and Prevention, Atlanta, GA; ${ }^{16}$ Sleep Disorders Institute, New York, NY; ${ }^{17}$ American Academy of Sleep Medicine, Darien, IL; *attendance by these individuals does not constitute endorsement of this statement by their affiliated institutions or organizations

Sleep is essential for optimal health. The American Academy of Sleep Medicine (AASM) and Sleep Research Society (SRS) developed a consensus recommendation for the amount of sleep needed to promote optimal health in adults, using a modified RAND Appropriateness Method process. The recommendation is summarized here. A manuscript detailing the conference proceedings and evidence supporting the final recommendation statement will be published in SLEEP and the Journal of Clinical Sleep Medicine.

Citation: Watson NF, Badr MS, Belenky G, Bliwise DL, Buxton OM, Buysse D, Dinges DF, Gangwisch J, Grandner MA, Kushida C, Malhotra RK, Martin JL, Patel SR, Quan SF, Tasali E. Recommended amount of sleep for a healthy adult: a joint consensus statement of the American Academy of Sleep Medicine and Sleep Research Society. SLEEP 2015;38(6):843-844.

\section{CONSENSUS STATEMENT}

- Adults should sleep 7 or more hours per night on a regular basis to promote optimal health.

- Sleeping less than 7 hours per night on a regular basis is associated with adverse health outcomes, including weight gain and obesity, diabetes, hypertension, heart disease and stroke, depression, and increased risk of death. Sleeping less than 7 hours per night is also associated with impaired immune function, increased pain, impaired performance, increased errors, and greater risk of accidents.

- Sleeping more than 9 hours per night on a regular basis may be appropriate for young adults, individuals recovering from sleep debt, and individuals with illnesses. For others, it is uncertain whether sleeping more than 9 hours per night is associated with health risk.

- People concerned they are sleeping too little or too much should consult their healthcare provider.

\section{METHODOLOGY}

Healthy sleep requires adequate duration, good quality, appropriate timing and regularity, and the absence of sleep disturbances or disorders. Sleep duration is the most frequently investigated sleep measure in relation to health. Furthermore,

\section{Submitted for publication April, 2015}

\section{Accepted for publication April, 2015}

Address correspondence to: Nathaniel F. Watson, MD, MSc; $2510 \mathrm{~N}$. Frontage Road, Darien, IL 60561; Tel: (630) 737-9700; Fax: (630) 7379790; Email: research@aasmnet.org. the US Department of Health and Human Services created a Sleep Health Objective to "increase the proportion of adults who get sufficient sleep" in Healthy People 2020, a federal initiative to improve the nation's health. ${ }^{1}$ Thus our recommendation focuses on this sleep parameter. A panel of 15 experts in sleep medicine and research used a modified RAND Appropriateness Method $^{2}$ to develop a recommendation regarding the sleep duration, or sleep duration range, that promotes optimal health in adults aged 18 to 60 years.

The expert panel reviewed published scientific evidence addressing the relationship between sleep duration and health, using a broad set of National Library of Medicine Medical Subject Headings (MeSH) terms and no date restrictions, which resulted in a total of 5,314 scientific articles. The process was further guided by the Oxford grading system. ${ }^{3}$ The panel focused on nine health categories with the best available evidence in relation to sleep duration: general health, cardiovascular health, metabolic health, mental health, immunologic function, human performance, cancer, pain, and mortality. Consistent with the RAND Appropriateness Method, multiple rounds of evidence review, discussion, and voting were conducted to arrive at the final recommendation. The process to develop this statement was conducted over a 12-month period and concluded with a consensus meeting held in February 2015 in Chicago, Illinois.

\section{DISCUSSION \& FUTURE DIRECTIONS}

Current evidence supports the general recommendation for obtaining 7 or more hours of sleep per night on a regular basis to promote optimal health among adults aged 18 to 60 years. Individual variability in sleep need is influenced by genetic, 
behavioral, medical, and environmental factors. A clearer understanding of the precise biological mechanisms underlying sleep need continues to require further scientific investigation.

This recommendation creates a foundation to raise awareness and improve understanding of sleep effects on health. The recommendation provides a basis for:

- Educating the public and healthcare providers on the importance of adequate sleep duration for health.

- Encouraging individuals to obtain adequate sleep duration.

- Discussing the economic and social benefits of adequate sleep duration, thereby informing public policy.

- Promoting research on the role of sleep duration in health and well-being.

Research that directly examines the effects of sleep duration on health may lead to revisions of this recommendation in the future.

\section{DISCLOSURE STATEMENT}

Funding for this project was provided by the American Academy of Sleep Medicine and Sleep Research Society, and by the cooperative agreement number 1U50DP004930-01 from the Centers for Disease Control and Prevention (CDC). Its contents are solely the responsibility of the authors and do not necessarily represent the official views of the CDC.

\section{REFERENCES}

1. US Department of Health and Human Services. Sleep Health Objectives. March 6, 2015. Available from: https://www.healthypeople.gov/2020/ topics-objectives/topic/sleep-health/objectives.

2. Fitch K, Bernstein SJ, Aguilar MD, et al. The RAND/UCLA Appropriateness Method User's Manual. Santa Monica, CA: RAND, 2001.

3. OCEBM Levels of Evidence Working Group. The Oxford Levels of Evidence 2. [cited February 9, 2015]. Available from: http://www.cebm. net/wp-content/uploads/2014/06/CEBM-Levels-of-Evidence-2.1.pdf. 